\title{
A High Efficiency 6LoWPAN Hierarchical Routing Protocol for Wireless Sensor Networks
}

\author{
Yukun Yao, Yaorui Liu* and Dongliang Xu \\ Key Lab of Mobile Communication Technology, Chongqing University of Posts \\ and Telecommunications, Chongqing, 400065, China \\ liuyaorui@foxmail.com
}

\begin{abstract}
IPv6 over low-power wireless personal area network (6LoWPAN) is proposed by IETF 6LoWPAN working group as a standard for IPv6 data transmission in wireless personal area network. In this paper, a high efficiency 6LoWPAN hierarchical routing protocol for wireless sensor networks(HE-HiLow) is proposed to solve the problems that the parent node selected by the subsequent is unreasonable and the path recovery is not resolved in the hierarchical routing over 6LoWPAN (HiLow), HE-HiLow protocol proposes a new parent node selection mechanism to ensure the selected parent more optimize and maintain the network load balancing by reducing control message redundancy and increasing the selection of parameters. Meanwhile, it also proposes a new path prejudge recovery mechanism to make some descendant nodes of the failed nodes to rejoin the network and continue to forward data in the uplink and downlink transmission process by prejudging the relationship between the failed node and itself. Simulation results show that, HE-HiLow protocol contributes to the balance of network load, the extension of network lifetime and the decrease of the average end-to-end delay.
\end{abstract}

Keywords: 6LoWPAN; HiLow; Parent node; Path recovery

\section{Introduction}

IPv6 over low-power wireless personal area network (6LoWPAN) is proposed by IETF 6LoWPAN working group as a standard for IPv6 data transmission in wireless personal area network. It can transmit IPv6 data packets in low-speed wireless networks. Since the publication of the related standard protocols, it has attracted wide attention from industry and academia. The standard of IPv6 packet transmission in low-power wireless personal area network is given, including the compression of IPv6 packet header, the reassembly of data, the configuration of address and so on. After depth research in recent years, now the fragmentation and reassembly of packets, the network management, the header compression and other aspects of technology have been a considerable progress in 6LoWPAN [1-3].

However, there is little research on the routing algorithm for 6LoWPAN. Kim et al., [4] modified the AODV protocol to avoid the network resource competitions in 6LoWPAN, but as they are still based on broadcast routing lookup mechanism, the network consumptions are huge, which are not fit for the 6LoWPAN low power restrictions. Felsche et al., [5] proposed a hierarchical routing protocol for 6LoWPAN. However, when the sensor network scales up, the address allocation will exacerbate the performance of the routing algorithm. The 6LoWPAN ad hoc on-demand distance vector routing [6] is a simplified on-demand routing protocol based on AODV [7] and is one of the most promising ones according to the activity related to this protocol within the 6LoWPAN. The author designed an alternate path mechanism to solve the path failure, but it does not meet the situations of mass of full function devices out of order in sudden. 
The hierarchical routing over 6LoWPAN [8] protocol is the most representative hierarchical routing protocols in 6LoWPAN, but HiLow protocol only defines address allocation algorithm and routing algorithm within a 6LoWPAN network without solving the interoperability issues with the external Internet IPv6 networks. Liu et al., [9] proposes three improvements on hierarchical routing of 6LoWPAN networks based on HiLow, with the consideration of practical application scenarios; this paper altered the normative address allocation method and modified mapping table in gateway, which makes the external Internet able to access 6LoWPAN network nodes, but this algorithm will have a huge consumption of address allocation. Zhao et al., [10] proposes a parent node selection strategy with average residual energy base on HiLow, but it can't maintain the balance of network load. Yue et al., [11] proposes an extended hierarchical routing protocol in 6LoWPAN(E-HiLow), this paper considers the node depth, residual energy and the number of node's child in the parent selection process, and selects the node with most average remaining energy and minimum depth to be its parent node, but it generates control message redundancy and also increases the time which was required for the node to select the parent node. Point to the problem mentioned above, this paper proposed an HE-HiLow protocol. This protocol can make more accurate to join nodes choose the parent node, effectively improves the network load balance ability, reduces the network control overhead, extends the network survival time and so on.

The rest of the paper is organized as follows. Section 2 presents a brief introduction to the traditional HiLow protocol. Section 3 shows problem description. Section 4 describes the proposed HE-HiLow in detail. Section 5 shows the simulation and performance evaluation, while conclusions are provided in section 6 .

\section{HiLow Protocol Overview}

All the nodes in the HiLow algorithm are distributed in tree form, and the data is forwarded according to the pre-defined dynamic address allocation algorithm, which avoids the overhead of routing table establishment and maintenance, and reduces the mutual signaling. The following is mainly about the dynamic address allocation algorithm and the routing process.

\subsection{Address Allocation Algorithm}

HiLow protocol dynamically configures the 16-bit short address for the node to initialize the allocation of address. When an IEEE 802.15.4 device wants to join a 6LoWPAN network, it first checks whether there is a 6LoWPAN network within its communication range. If the node doesn't detect network, the node will automatically act as a new coordinator node and assign a short address of value is 0 for itself; if the detection is successful, the node associated with the parent node and can receive a 16-bit short addresses from the parent node. The formula of address assignment as follows:

$$
F C=M C * A P+N(0<N<=M C)
$$

In Eq. (1), where $M C$ is the maximum number of child nodes that a parent can have, $A P$ is the address of parent node, $N$ is the $N$-th child node of parent node.

\subsection{Routing Operations}

To transmit a packet, HiLow protocol assumes that every node knows its own depth of the routing tree that nodes can directly compute the address of next hop. Similarly, when the relaying node receives a packet from a source node, it obtains its own parent node address as follows: 


$$
P C=\lfloor(C-1) / M C\rfloor
$$

In Eq. (2), where $P C$ is the address of parent, $C$ is the address of the current node which has received a packet, and $L\rfloor$ is the symbol of the floor operation. The current node can determine whether it is the ascendant or the descendant node of the destination by using this formula. When the current node receives a packet, the next hop node to forward the packet can be calculated by the following three basic cases $(A A(m, Y)$ denotes the address of the ascendant node of depth $m$ of the node $Y, d$ is the depth of the current node):

(i) If the current node $C$ is one of the ascendant nodes of the destination $D$, the next hop is $A A(d+1, D)$.

(ii) If the current node $C$ is one of the descendant nodes of the destination $D$, the next hop node is $A A(d-1, C)$, the parent node of $C$, which can be calculated by Eq. (2).

(iii) Otherwise, the next hop is $A A(d-1, C)$.

\section{Problem Description}

To simplify the description of the problem, this paper first gives a simplified example of parent node selection, as shown in Figure 1.

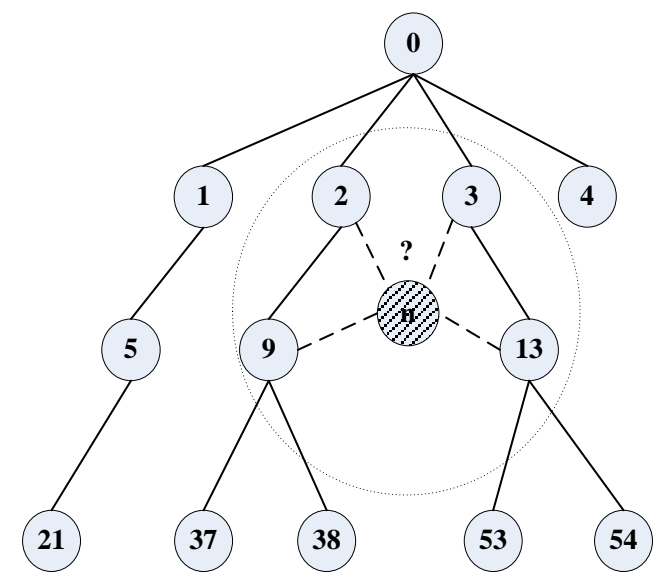

Figure 1. Example of Parent Node Selection

In order to clearly describe the E-HiLow protocol in the specific problems, this paper gives the following definitions:

Definition 1: Uplink transmission process, which represents the process that a packet is transmitted by the child node to the parent node.

Definition 2: Downlink transmission process, which represents the process that a packet is transmitted by the parent node to the child node.

The E-HiLow protocol [11] proposes address allocation strategy and path recovery strategy, it can make the potential parent nodes respond the request within its address, current number of child node, remaining energy and so on. Then the new node could find out the parent node with least overhead, which will certainly balance the routing hierarchical tree. But there are two problems in the protocol:

(1) In the process of selecting the parent node, the potential parent nodes respond the request within its address, current number of child node, remaining energy and so on. The weight value of each potential parent node is sequentially calculated by the network request node according to the weight formula and the parent node is selected according to 
the weight criteria. But this process generates control message redundancy and also increases the time which was required for the node to select the parent node.

(2) In the process of path recovery, there is no solution to the problem of downlink path recovery. During data forwarding, a node can only communicate with its parent node or child node, if a node does not communicate with its child node during the downlink transmission, and the route is not repaired, the failed node will affect the communication between other nodes.

\section{HE-HiLow Protocol}

In this paper, we propose an improved HiLow protocol named HE-HiLow based on EHiLow. The main contribution of HE-HiLow is designed two new mechanisms: (1) Parent node selection mechanism. (2) Path prejudge recovery mechanism.

\subsection{Parent Node Selection Mechanism}

The steps of parent node selection mechanism are as follows:

Step 1: When a new node joins into 6LoWPAN networks, it firstly monitor whether there has been a 6LoWPAN network by its scanning procedure. If not, it allocates the address 0 to itself, becomes the network coordinate node in 6LoWPAN; if it discovers just one coordinate node, then puts $\mathrm{J}$ (Join) with $1, \mathrm{R}$ (Recovery) with 0 , M (Multi) with 0 , sends "Join Request" to the coordinate node, which will associate in MAC layer, shown in Figure 2; if it finds more than one coordinate node, puts $\mathrm{J}$ with 1 , $\mathrm{R}$ with 0 , $\mathrm{M}$ with 1 , broadcasts the "Join Request" message;

\begin{tabular}{|ll|lllllll|l}
$\mathbf{0}$ & $\mathbf{1}$ & $\mathbf{2}$ & $\mathbf{3}$ & $\mathbf{4}$ & $\mathbf{5}$ & $\mathbf{6}$ & $\mathbf{7}$ & $\mathbf{8}$ & $\mathbf{9}$ \\
\hline $\mathbf{1}$ & $\mathbf{1}$ & $\mathbf{0}$ & $\mathbf{0}$ & $\mathbf{0}$ & $\mathrm{J}$ & $\mathbf{R}$ & $\mathbf{M}$ & & \\
\hline
\end{tabular}

Figure 2. Request Message Format

Step 2: When a coordinate node $i$ receives a "Join Request", it firstly checks the number of child node $m_{i}$, if $m_{i}$ is equal to $M C$, which means the node is not suitable as the parent node, then ignores the "Join Request"; if $m_{i}$ is less than $M C$, then checks the M mark,

\begin{tabular}{|ll|lllllllllll|r}
$\mathbf{0}$ & 1 & 2 & 3 & 4 & 5 & 6 & 7 & 8 & 9 & 0 & 1 & 2 & 3 \\
\hline 1 & 1 & 0 & 0 & 1 & J & R & A & & Weight & & \\
\hline
\end{tabular}

Figure 3. Acknowledge Message Format

i). If $\mathrm{M}$ shows 0 , which means there is no other candidate parent node, then calculates the child node's address following Eq. (1), and the association is completed;

ii). If $\mathrm{M}$ shows 1 , that means more than one coordinate node received the "Join Request", then the node checks its remaining energy $P_{i}$ and depth $D_{i}$, if $P_{i} / m_{i}$ is less than the value of $L P E$, then ignores the "Join Request". Else the node $i$ calculates weight value $W_{i}$ according to the Eq. (3), and packets its $W_{i}$, puts J with 1 , R with 0, A (Acknowledge) with 1, sends "Join Acknowledge" to the request node, shown in Figure 3.

$$
W_{i}=\frac{P_{i}}{m_{i} *\left(M C^{D_{i}}\right)}
$$


Step 3: Supposing the request node receives $n$ "Join Acknowledge" messages from candidate parent nodes, and selects the node with the largest weight value to be its parent node $N_{\max }$ according to the Eq. (4):

$$
N_{\text {max }} \Leftarrow \max \left\{W_{1}, W_{2}, \mathrm{~L}, W_{n}\right\}
$$

\subsection{Path Prejudge Recovery Mechanism}

To simplify the description of the path prejudge recovery mechanism, this paper gives a simplified example of path recovery, as shown in Figure 4.

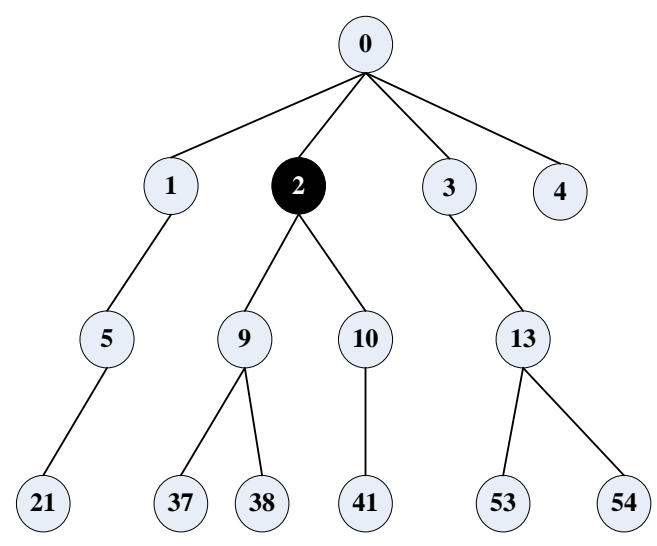

Figure 4. Example of Path Recovery

The steps of path prejudge recovery mechanism are as follows:

Step 1: In the process of data transmission, if node $y$ finds that its next hop node is the failed node, it will firstly judge the relationship between the failed node and itself. If the failed node is the parent of node $y$, then node $y$ puts $\mathrm{J}$ (Join) with $0, \mathrm{R}$ (Recovery) with 1 , $\mathrm{M}$ (Multi) with 1, and broadcasts the "Recovery Request" message with the failed node address; if the failed node is the child of node $y$, then node $y$ puts $\mathrm{J}$ with $1, \mathrm{R}$ with $1, \mathrm{M}$ with 1 , and broadcasts the "Recovery Request" message with the failed node address.

Step 2: When a coordinate node $i$ receives a "Recovery Request" message, the node $y$ will firstly judge whether the failed node is the parent of node $i$. If the failed node is the parent of node $i$, then node $i$ puts $\mathrm{J}$ with $0, \mathrm{R}$ with $1, \mathrm{M}$ with 1 , and broadcasts the "Recovery Request" message with the failed node address; if the failed node is not the parent of node $i$, node $i$ will check the $\mathrm{J}$ and $\mathrm{R}$ mark:

i). If $\mathrm{J}$ shows 1 and $\mathrm{R}$ shows 1 , node $i$ ignores the "Recovery Request" message;

ii). If $\mathbf{J}$ shows 0 and $\mathrm{R}$ shows 1 , node $i$ checks the number of child node $m_{i}$, if mi is equal to $M C$, which means the node is not suitable as the stepparent node, then ignores the "Recovery Request"; if $m_{i}$ is less than $M C$, node $i$ checks its remaining energy $P_{i}$ and depth $D_{i}$, if $P_{i} / m_{i}$ is less than the value of $L P E$, then ignores the "Recovery Request". Else the node calculates weight value $W_{i}$ according to the Eq. (3), and packets its $W_{i}$, puts $\mathbf{J}$ with $1, \mathrm{R}$ with 0 , A (Acknowledge) with 1, sends "Join Acknowledge" to the request node.

Step 3: Supposing the request node receives $n$ "Join Acknowledge" messages from candidate stepparent nodes, and selects the node with the largest weight value to be its stepparent node $N_{\max }$ according to the Eq. (4). 
Step 4: The repaired node should update its descendent 16-bit short address level by level. Supposing the old address of parent node is $A P_{\text {old }}$, the new address of parent node is $A P_{n e w}$, and the maximum number of child node is $M C$, then we can get the new address of child node $A C_{\text {new }}$ by its old address $A C_{\text {old }}$ using the Eq. (5):

$$
A C_{\text {new }}=\left(A P_{\text {new }}-A P_{\text {old }}\right) * M C+A C_{\text {old }}
$$

\section{Simulation and Performance Evaluation}

In this paper, we use the OPNET14.5 platform to build the simulation platform, and select the HiLow protocol and E-HiLow protocol as the comparison object. Through the comparison of simulation result, we can analysis the differences about the nodes survival ratio, the average end-to-end delay for the three protocols.

\subsection{Network Scene and Parameter}

In order to compare with the E-HiLow protocol of [11], we also set up a low-power, multi-hop sensor network within $150 \mathrm{~m} \times 150 \mathrm{~m}$ area, and increase the node number step by step. Specific simulation parameters are shown in Table 1.

\section{Table 1. The Specific Simulation Parameters}

\begin{tabular}{|l|l|}
\hline Parameter & Value \\
\hline Simulation duration & $800 \mathrm{~s}$ \\
\hline Number of nodes & 60 \\
\hline Simulated area & $150 \mathrm{~m} \times 150 \mathrm{~m}$ \\
\hline Transmission rate & $200 \mathrm{kbps}$ \\
\hline Initial energy of nodes & $20 \mathrm{~J}$ \\
\hline Transmission distance & $10 \mathrm{~m}$ \\
\hline MAC layer & 802.15 .4 \\
\hline
\end{tabular}

\subsection{Simulation Results and Analysis of Each Performance Index}

(1) The Nodes Survival Ratio: It denotes the rate of the number of nodes which can effectively transmit data and the total number of nodes in the network, the calculation formula is as follows:

$$
T=\frac{N-N_{d i e}}{N}
$$

In Eq. (6), $T$ is the nodes survival ratio, and $N$ is the total number of nodes in the network, $N_{\text {die }}$ is the number of failed node in the network. 


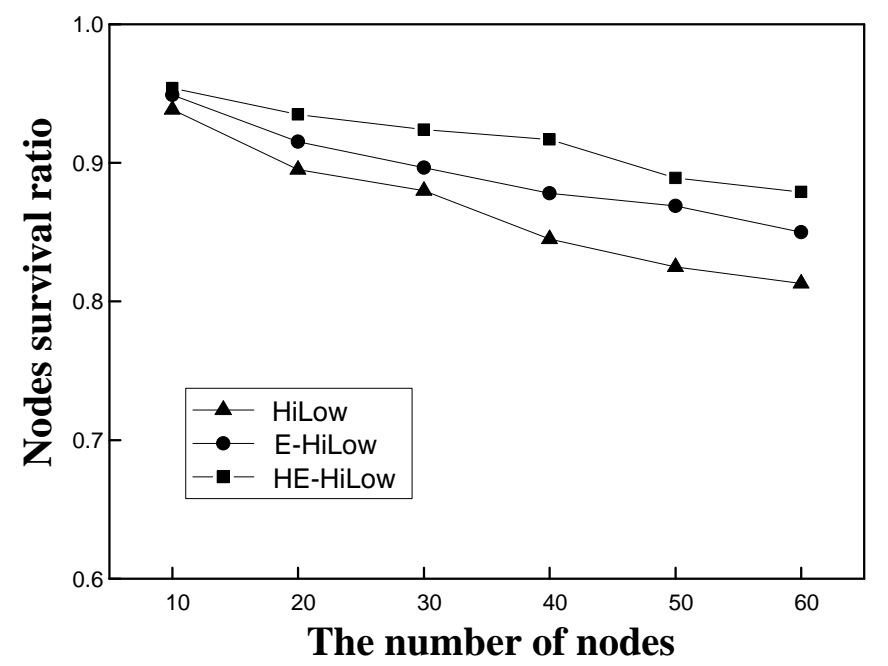

Figure 5. The Nodes Survival Ratio

Figure 5 shows the nodes survival ratio of HiLow, E-HiLow, and HE-HiLow three protocols. From Figure 4 we can see that three protocols all show a down trend with the increasing number of nodes. However, HiLow protocol first appears the failed node and the decline range of curve is the largest. E-HiLow protocol proposes address allocation strategy and path recovery strategy, so it later appears the failed node and the decline is the smaller. HE-HiLow protocol reduces the cost of the control message in the selection of the parent node and considers the order of arrival of the control message. This ensures that the selected parent node is more optimized and it will achieve the load balance. On the other hand, HE-HiLow protocol proposes path prejudge recovery mechanism, it can let the failed node rejoin the network, and prolong the lifetime of network more effectively. So HE-HiLow protocol appears the failed node at the latest and the decline is the smallest.

(2) The Average End-to-End Delay: It denotes the average delay of all packets from the source node to the destination node, the calculation formula is as follows:

$T_{\text {avg }}=\frac{\sum_{i=1} T_{i}}{N_{\text {num }}}$

In Eq. (7), $T_{\text {avg }}$ is the average end-to-end delay, and $T_{i}$ is the delay of the $i$-th packet from the source node to the destination node, $N_{\text {num }}$ is the total number of packets that have reached the destination node. 


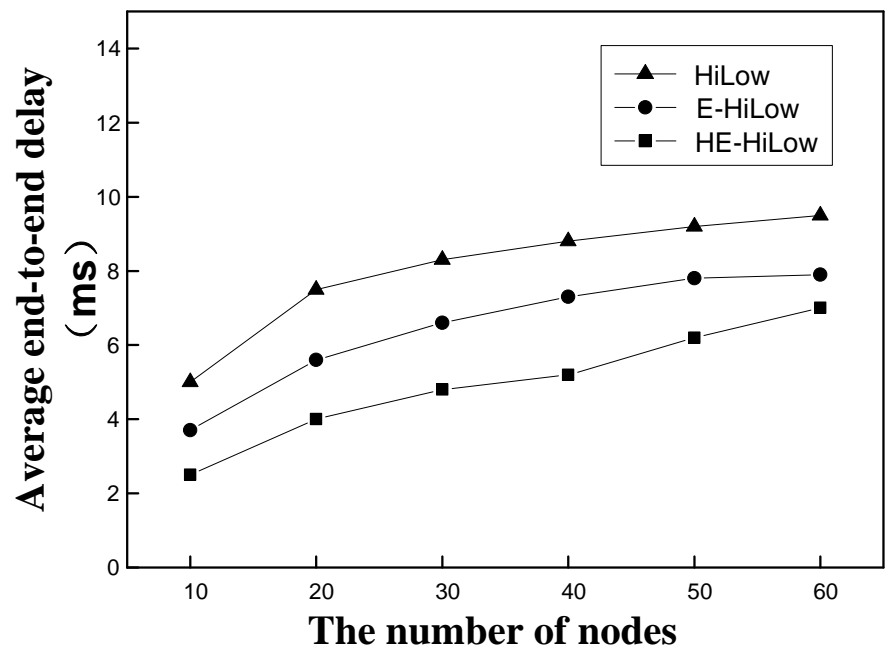

Figure 6. The Average End-to-End Delay

Figure 6 shows the average end-to-end delay of HiLow, E-HiLow, and HE-HiLow three protocols. From Figure 6 we can see that three protocols all show a rising trend with the increasing number of nodes. However, the range of HiLow protocol is the greatest, this is due to the load imbalance of its hierarchical routing tree. The range of E-HiLow protocol is the smaller than HiLow, the reason is that E-HiLow protocol select the node which has the maximum average remainder energy and minimum depth as the parent node, and its load will be more balanced than HiLow protocol. HE-HiLow protocol proposes the parent node selection mechanism, this mechanism reduces the time which was required for some descendant nodes of the failed node to select the step-parent node in the path recovery process, so the rising range of HE-HiLow protocol is the smallest.

\section{Conclusion}

This paper presents a high efficiency 6LoWPAN hierarchical routing protocol for wireless sensor networks (HE-HiLow), this protocol proposes two new mechanisms to solve the problem of E-HiLow protocol. Firstly, it uses the parent node selection mechanism to ensure that the selected parent is more optimized, the load balance of the network is maintained and the average end-to-end delay is reduced. Finally, it uses the path prejudge recovery mechanism to make some descendant nodes of the failed nodes to rejoin the network and continue to forward data in the uplink and downlink transmission process, and it improves the network lifetime.

\section{Acknowledgment}

Our research is partly supported by the project of the National Natural Science Foundation of China (No. 61379159).

\section{References}

[1] J. W. Hui, and D. E. Culler, "Extending IP to Low-Power, Wireless Personal Area Networks", IEEE Internet Computing, vol. 24, no. 11, (2008), pp. 37-45.

[2] J. P. Sheu, S. C. Tu, and L. H. Chan, "A Distributed IP Address Allocation Scheme in Ad Hoc Networks", International Journal of Ad Hoc and Ubiquitous Computing, vol. 3, no. 1, (2007), pp. 10-20.

[3] H. Shin, E. Talipov and H. Cha, "Spectrum: Lightweight Hybrid Address Autoconfiguration Protocol Based on Virtual Coordinates for 6LoWPAN", IEEE Transactions on Mobile Computing, vol. 11, no. 11, (2012), pp. 1749-1762. 
[4] E Kim, D Kaspar, and C Gomez, "Problem Statement and Requirements for IPv6 over Low-Power Wireless Personal Area Network (6LoWPAN) Routing”, Heise Zeitschriften Verlag, vol. 15, no. 4, (2012), pp. 26-30.

[5] M Felsche, and A Huhn, Editor, Routing Protocols for 6LoWPAN, IT Revolutions, Springer Berlin Heidelberg, (2011).

[6] J. M. Chang, H. Y. Yang, and H. C. Chao, Multipath Design for 6LoWPAN Ad Hoc on-demand Distance Vector Routing, International Journal of Information Technology Communications and Convergence, vol. 1, no. 1, (2010), pp. 24-40.

[7] F Yang, and B Sun, Ad Hoc on-demand Distance Vector Multipath Routing Protocol with Path Selection Entropy, Consumer Electronics, Communications and Networks (CECNet), 2011 International Conference on IEEE, Xianning, China, (2011) April, 4715-4718.

[8] Oliveira, and Lui, Routing and Mobility Approaches in IPv6 over LoWPAN Mesh Networks, International Journal of Communication Systems, vol. 24, no. 11, (2011), pp. 1445-1466.

[9] M Liu, Y Xu, and S Wu, Design and Optimization of Hierarchical Routing Protocol for 6LoWPAN, International Journal of Distributed Sensor Networks, vol. 2015, no. 13, (2015), pp. 1-10.

[10] Y Zhao, F Li, and Z. P. Jia, Extended Hierarchical Routing Protocol in 6LoWPAN Networks, Computer Engineering and Applications, vol. 47, no. 7, (2011), pp. 78-81.

[11] L Yue, D Xie, and J Zhao, E-HiLow: Extended Hierarchical Routing Protocol in 6LoWPAN Wireless Sensor Network, IEEE Eighth International Conference on Networking, Architecture and Storage, IEEE Computer Society, Xian, China, (2013) July 8-12.

\section{Authors}

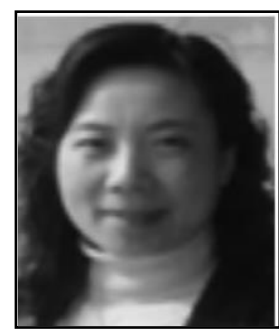

Yukun Yao, is a Professor from Chongqing University of Posts and Telecommunications. Her research interests are: network coding, network management and application.

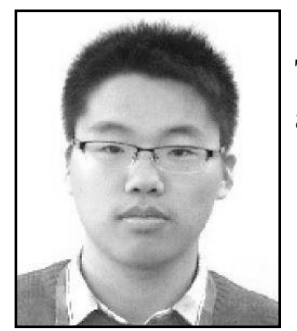

Yaorui Liu, is a master from Chongqing University of Posts and Telecommunications, year in 2014 and now. His research interests are: wireless sensor networks, and the Internet of Things (IoT). 
International Journal of Advanced Science and Technology Vol.99 (2017) 